\title{
Transdermal Evaluation of Caffeine in Different Formulations and Excipients
}

\author{
Lieselotte Veryser, ${ }^{1}$ Jente Boonen, ${ }^{1}$ Els Mehuys, ${ }^{2}$ Nathalie Roche, ${ }^{3}$ Jean-Paul Remon, ${ }^{2}$ \\ Kathelijne Peremans, ${ }^{4}$ Christian Burvenich, ${ }^{4}$ and Bart De Spiegeleer ${ }^{1}$
}

Background: The stratum corneum (SC) forms a difficult physical barrier for drugs to pass through the skin. Several strategies were developed to overcome this barrier. Optimization of topical drug formulations by selected excipients may facilitate the penetration of drugs through the SC into the viable skin cells and ultimately into the systemic circulation. Methods: Here, both the influence of two formulations (a classic carbomer-based gel and a novel Pluronic ${ }^{\circledR}$ lecithin organo gel (PLO gel)) and selected excipients (ethanol, propylene glycol, diethylene glycol monoethyl ether, isopropyl myristate (IPM), and water) with or without the penetration enhancer miconazole nitrate on the transdermal penetration characteristics of caffeine were determined using an in vitro Franz diffusion cell setup.

Results: Higher fluxes were observed for the carbomer-based gel compared to the PLO gel. Among the commonly used excipients, IPM showed the best penetration enhancing properties, while the presence of the penetration enhancer miconazole nitrate did not significantly alter the apparent skin permeation characteristics for caffeine.

Conclusion: The high ethanol percentage in the carbomer-based gel could explain the results as supported by our excipient data. Moreover, IPM could play a beneficial role in topical formulations as this excipient was responsible for a significant increase in the amount of caffeine penetrated through the skin. No overall statistical significant effect of the presence of miconazole nitrate as a penetration enhancer was observed.

\section{Introduction}

$\mathbf{T}$ HE SKIN As a route for drug administration has become very attractive. Transdermal drug delivery offers several other advantages in comparison with the traditional oral route of administration, such as minimization of the first pass effect and avoidance of the acidic environment of the stomach, which could be responsible for drug degradation. This results in a higher bioavailability of the drug, decreased side effects, and more stable plasma levels. Transdermal drug delivery also provides more controlled long-term drug delivery ${ }^{1,2}$ and represents a noninvasive route of administration, which can result in improved patient adherence. ${ }^{3}$

Topical administration of caffeine can be used both for local as well as for a systemic activity. Moreover, the internationally accepted guidelines 427 and 428 from the Organization for Economic Co-operation and Development (OECD) recommend the use of caffeine as a model compound in skin research., ${ }^{4,5}$

Skin transport of drugs can be investigated using in vivo studies with humans and are considered to be the gold standard. However, for ethical reasons, the ex vivo/in vitro meth- ods, using excised human or animal skin are more frequently used. The skin can be seen as a homogeneous brick and mortar model (i.e., stratum corneum (SC) corneocytes with surrounding lipids) with an underlying viable epidermis consisting of different strata and the dermis. Herein, the SC is a difficult barrier to overcome. Optimization of the topically applied formulation can improve the release of the active drug to and through the skin. A classic strategy for modifying the skin barrier function is the use of selected excipients. The aim of this study was to examine whether the two topical formulations and selected topical excipients, with or without the recently discovered penetration enhancer miconazole nitrate, ${ }^{6}$ have an influence on the transdermal behavior of caffeine.

\section{Materials and Methods}

\section{Products examined}

Caffeine (Ph. Eur. grade) was obtained from ABC Chemicals (Vemedia, Wouters-Brakel, Belgium) or from Ludeco (Sint-Gillis, Belgium). Miconazole nitrate (MN) was also bought from ABC Chemicals (Vemedia, Wouters-Brakel,

\footnotetext{
${ }^{1}$ Drug Quality and Registration (DruQuaR) group, Faculty of Pharmaceutical Sciences, Ghent University, Ghent, Belgium.

${ }^{2}$ Laboratory of Pharmaceutical Technology, Faculty of Pharmaceutical Sciences, Ghent University, Ghent, Belgium.

${ }^{3}$ Department of Plastic and Reconstructive Surgery, Ghent University Hospital, Ghent, Belgium.

${ }^{4}$ Department of Comparative Physiology and Biometrics, Faculty of Veterinary Medicine, Ghent University, Merelbeke, Belgium.
} 
Belgium). The Pluronic ${ }^{\circledR}$ lecithin organo gel and the carbomerbased gel were prepared by the Laboratory of Pharmaceutical Technology (Faculty of Pharmaceutical Sciences, Ghent, Belgium). The PLO gel consisted of $1 \mathrm{~g}$ of caffeine, $19 \mathrm{~g}$ of the lecithin solution (Fagron, Waregem, Belgium; content: lecithin, isopropyl palmitate, and potassium sorbate), and $80 \mathrm{~g}$ of the Pluronic ${ }^{\circledR}$ gel (Fagron, Waregem, Belgium; content: Pluronic F127 (Poloxamer $407^{\circledR}$ ), potassium chloride, potassium sorbate, sodium benzoate, and cold water), while the carbomer gel consists of $1 \mathrm{~g}$ of caffeine, $12 \mathrm{~g}$ of propylene glycol (PG), $3 \mathrm{~g}$ of carbomer 980 , and $84 \mathrm{~g}$ of ethanol $(96 \% \mathrm{~V} / \mathrm{V})$.

\section{Chemicals and reagents}

About $0.01 \mathrm{M}$ phosphate-buffered saline (PBS) was prepared according to the instructions of the supplier (Sigma, St. Louis, MO). Ultrapure water $\left(\mathrm{H}_{2} \mathrm{O}\right)$ of $18.2 \mathrm{M} \Omega$.cm quality was obtained by using an Arium 611 purification system (Sartorius, Göttingen, Germany). High-performance liquid chromatography (HPLC), gradient grade methanol (MeOH), and acetonitrile $(\mathrm{ACN})$ were purchased from Fisher Scientific (Leicestershire, United Kingdom). Formic acid (FA) and PG were obtained from Riedel-de Haën (Seelze-Hannover, Germany). Denaturated ethanol (up to $5 \%$ ether) was obtained from Chem Lab (Zedelgem, Belgium), while absolute ethanol $(\mathrm{EtOH}, 99.8 \% \mathrm{~V} / \mathrm{V})$ was obtained from Sigma. Diethylene glycol monoethyl ether (DGME) and isopropyl myristate (IPM) were bought from Fluka (Steinheim, Germany) and hydrogen chloride $(\mathrm{HCl})$ from Acros organics (Geel, Belgium).

\section{Preparations of saturated solutions and solubility determination}

An excess amount of caffeine $(n=2-3)$ was added to each excipient (EtOH, PG, DGME, IPM, $\mathrm{H}_{2} \mathrm{O}, 50: 50$ EtOH: $\mathrm{H}_{2} \mathrm{O}$ ) and subsequently incubated for 24 hours at $32^{\circ} \mathrm{C}$ (skin surface temperature $^{4}$ ), while shaking on a laboratory Eppendorf Thermo mixer at $1000 \mathrm{rpm}$. Vortex mixture was done at regular time points. After incubation, the samples were centrifuged with an Eppendorf 5417R centrifuge (Hamburg, Germany) for 10 minutes at 20,000 $g$, while keeping the temperature at $32^{\circ} \mathrm{C}$. The clear supernatant was collected and immediately diluted with the appropriate mobile phase (20:80 $\left.\mathrm{ACN}: \mathrm{H}_{2} \mathrm{O}(\mathrm{V} / \mathrm{V})\right)$. The concentration of caffeine was determined in the resulting solution using a validated HPLC-UV method (see 2.5.). Due to incompatibility of IPM with the HPLC mobile phase, solubility data of caffeine in IPM were obtained from UV/VIS spectra at $273 \mathrm{~nm}$ (Ultrospec 4000 Pharmacia Biotech UV/VIS spectrophotometer, England).

\section{Preparation of Franz diffusion cell dose solutions}

The gel formulations. To investigate the effect of the used formulation on the transdermal behavior of caffeine, $600 \mathrm{mg}$ of the PLO gel and carbomer-based gel, each containing $1 \%$ caffeine, were topically applied to the epidermal surface of the skin in a Franz diffusion cell (FDC) setup.

Caffeine dose solutions in different excipients. Dose solutions were prepared with caffeine to obtain a $2 \mathrm{mg} / \mathrm{mL}$ caffeine solution with or without $1.0 \times 10^{-5} \mathrm{M}$ miconazole nitrate. $^{6}$ Due to limited solubility of caffeine in IPM, a $0.4 \mathrm{mg} / \mathrm{mL}$ caffeine $( \pm \mathrm{MN})$ IPM-dose solution was prepared.

\section{High-performance liquid chromatography}

Caffeine in the different FDC samples was analyzed using a validated high-throughput HPLC-UV method. The HPLC apparatus consisted of a Waters Alliance 2695 separation module and a dual absorbance detector 2487 equipped with Empower 2 software (all Waters, Millford, PA). About $20 \mu \mathrm{L}$ of each sample was injected on a Lichrospher 100 RP C18 column $(125 \mathrm{~mm} \times 4 \mathrm{~mm}, 5 \mu \mathrm{m}$ particle size) (Merck, Darmstadt, Germany) with an appropriate guard column. The sample compartment was kept constant at $15^{\circ} \mathrm{C}$, while the column temperature was maintained at $30^{\circ} \mathrm{C}$. The flow rate was set at $1 \mathrm{~mL} / \mathrm{min}$. A degassed isocratic mobile phase consisting of $83: 17 \mathrm{~A}: \mathrm{B}\left(\mathrm{V} / \mathrm{V}, \mathrm{A}: 0.1 \% \mathrm{FA}\right.$ in $\mathrm{H}_{2} \mathrm{O}, \mathrm{B}: 0.1 \% \mathrm{FA}$ in $\left.\mathrm{ACN}\right)$ or $75: 25 \mathrm{~A}: \mathrm{C}(\mathrm{V} / \mathrm{V}, \mathrm{C}: 0.1 \% \mathrm{FA}$ in $\mathrm{MeOH})$ were used for the caffeine determination in the excipients and gels experiment, respectively. UV detection was performed at $273 \mathrm{~nm}$.

\section{In vitro permeation study}

The skin permeation of caffeine in the different dose formulations was determined using static FDCs (Logan Instruments Corp.) with a receptor compartment of $5 \mathrm{~mL}$ and an available diffusion area of $0.64 \mathrm{~cm}^{2}$. The analyses were minimally done in duplicate using human skin in a randomized blocked design, that is, same skin donor sample (cut in several small FDC pieces) was used for the different formulations during one experimental run. The skin samples, supplied by the University Hospital (Ghent, Belgium), were obtained after a cosmetic reduction surgery from a healthy male patient (59 years) and from three healthy females ( $48 \pm 7$ years old, mean $\pm \mathrm{SD}$ ), to incorporate possible gender variability, $^{7-9}$ with informed consent and confidentiality procedures in place. Subsequently, the skin was cleaned with $0.01 \mathrm{M}$ PBS pH 7.4 and the subcutaneous fat was removed. The skin was then stored at $-20^{\circ} \mathrm{C}$ for not longer than 6 months. Just before the start of the FDC experiments, the full-thickness skin was thawed, mounted on a template, and dermatomed using an electrical powered dermatome to obtain a skin thickness of approximately $400 \mu \mathrm{m}$. An actual skin thickness of $380 \pm 11 \mu$ m (mean \pm SEM, $n=25$ ), $277 \pm 16 \mu \mathrm{m}$ (mean \pm SEM, $n=18), 421 \pm 19 \mu$ m (mean \pm SEM, $n=20$ ), and $368 \pm 27$ (mean \pm SEM, $n=20) \mu$ m was experimentally determined with a micrometer (Mitutoyo, Tokyo, Japan) from different patients. Each receptor chamber was filled with $0.01 \mathrm{M}$ PBS. The skin samples were visually inspected for skin damage and were mounted on the FDC between the donor and the receptor chambers, with the epidermis upward, taking care that no air under the skin was present. The receptor fluid was continuously mixed using a Teflon-coated magnetic stirring bar (400 rpm). Skin integrity was checked by measuring the skin impedance using an automatic microprocessor controlled Tinsley LCR Impedance Bridge (Croydon, United Kingdom). Skin pieces with an impedance value below $10 \mathrm{k} \Omega$ were discarded and replaced by a new skin piece. ${ }^{10}$ Approximately $600 \mathrm{mg}$ of the gels or $500 \mu \mathrm{L}$ of the excipient-dose solutions were topically applied on the skin surface with a syringe or micropipette, respectively. To prevent evaporation of the dose formulations, the donor chamber was covered with parafilm (American National $\mathrm{Can}^{\mathrm{TM}}$, Chicago, IL). During the experiment, the temperature of the receptor compartment was kept constant at $32^{\circ} \mathrm{C}$ by a water jacket. About $200 \mu \mathrm{L}$ samples of receptor fluid were 
taken at regular time intervals up to 24 hours from the sample port and were immediately replaced by $200 \mu \mathrm{L}$ fresh receptor fluid. This was taken into account in the cumulative concentration calculations. Immediately after the last sample had been drawn (i.e., after 24 hours), the remaining dose formulation was removed from the skin surfaces using a cotton swab and caffeine was extracted from the skin with ethanol. A linear relationship of the individual cumulative amounts of caffeine versus time was observed and steady-state and sink conditions were confirmed by our data.

\section{Calculation of skin permeation parameters}

The cumulative amount of caffeine (in $\mu \mathrm{g}$ ) permeated through human skin was plotted as a function of time (in hours). Steady-state flux $\left(\mathrm{J}_{\mathrm{ss}}, \mu \mathrm{g} / \mathrm{cm}^{2} . \mathrm{h}\right)$ was calculated from the slope of the linear portion of the cumulative amount versus time curve divided by 0.64 (exposed skin area in $\mathrm{cm}^{2}$ ). By setting $y=0$ in the individual linear regression equation, the lag time (h) was obtained. From these secondary parameters, the primary parameters are calculated in accordance with ECETOC, CEFIC ${ }^{11}$ : the permeability coefficient $\left(K_{p}\right.$, $\mathrm{cm} / \mathrm{h}$ ) could be calculated as follows:

$$
K_{p}=\frac{J_{s s}}{c_{d}}
$$

where $C_{d}(\mu \mathrm{g} / \mathrm{mL})$ is the concentration of caffeine in the dose formulation. Moreover, the apparent diffusion $\left(D_{m}, \mathrm{~cm}^{2} / \mathrm{h}\right)$ and partition $\left(\mathrm{K}_{\mathrm{m}}\right)$ coefficients are calculated using the following equations:

$$
\begin{gathered}
D_{m}=\frac{d^{2}}{6 \times t_{\text {lag }}} \\
K_{m}=\frac{K_{p} \times d}{D_{m}}
\end{gathered}
$$

where $d$ and $t_{\text {lag }}$ are the measured skin thickness $(\mathrm{cm})$ and the lag time (h), respectively.

\section{Results}

\section{Influence of the formulation on the transdermal} behavior of caffeine

The flux curves of caffeine in the carbomer and PLO gel are presented in Figure 1.

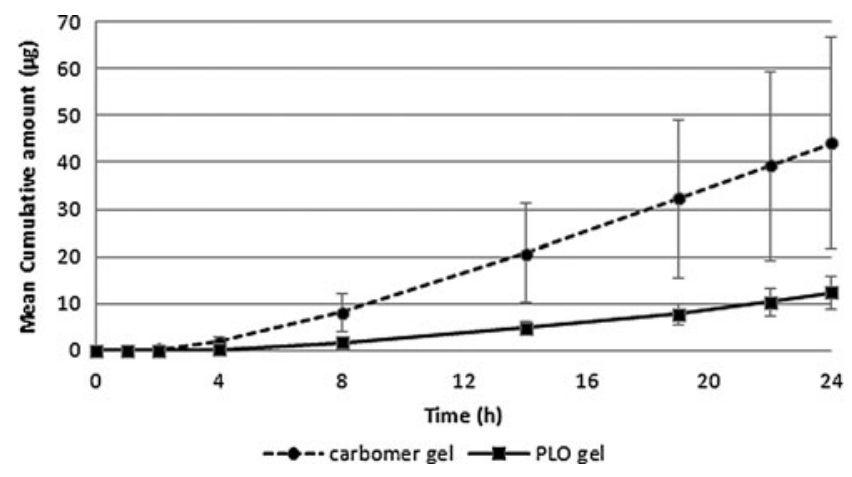

FIG. 1. Cumulative amount of drug in the receptor fluid after application of caffeine in the carbomer and PLO gel on isolated human skin $\left(n=3\right.$, mean \pm SEM). PLO, Pluronic ${ }^{\circledR}$ lecithin organo gel.

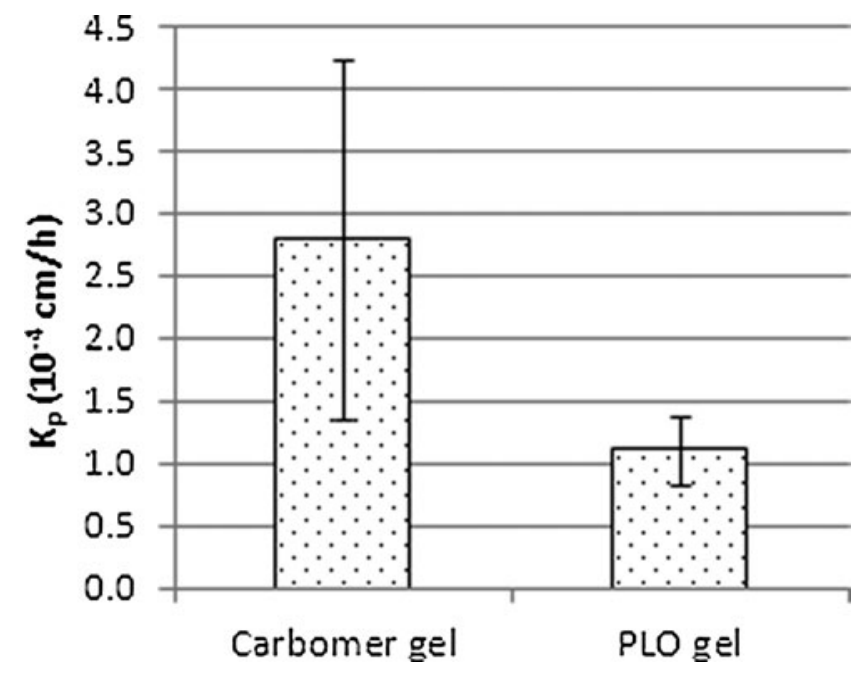

FIG. 2. Permeability coefficient of caffeine in the investigated gels $(n=3$, mean \pm SEM).

Secondary and primary transdermal parameters were calculated from the linear regression data. A higher flux and resulting permeability coefficient $\mathrm{K}_{\mathrm{p}}$ for caffeine was observed with the classic carbomer-based gel compared to the PLO gel (Fig. 2).

Not only the $\mathrm{D}_{\mathrm{m}}$ of caffeine formulated in the carbomer gel was higher than in the PLO gel (Fig. 3), indicating the formulation composition most probably changed the structure of the skin, but also the partitioning of caffeine from the carbomer gel was higher compared to the PLO gel.

\section{Solubility data of caffeine in the different excipients}

The solubility of caffeine at $32^{\circ} \mathrm{C}\left(\mathrm{S}_{32^{\circ} \mathrm{C}}\right)$ in the different excipients was determined in triplicate. The results are shown in Table 1.

\section{Excipient effects on the transdermal behavior of caffeine}

The primary transdermal parameters for caffeine solubilized in the different topical excipients $( \pm M N)$ are given in Table 2.

The choice of excipients had a major influence on the transdermal characterization of caffeine: in IPM, caffeine showed a significant $K_{p}$ increase compared to the other excipients (Table 2). While the diffusion coefficient obtained with IPM was comparable to the other $D_{m}$ values, the $K_{m}$ was noticeable higher, indicating that caffeine was more partitioned into the skin from the IPM dose solution compared to other excipients. Overall, no statistically significant effect of MN could be demonstrated on the $\mathrm{K}_{\mathrm{p}}$ of caffeine (paired $t$-test: $t=-0.791, \mathrm{df}=5,0.465$ significance), consistent with our previous observations, ${ }^{6}$ although small effects for individual excipients cannot be ruled out in the case of pure $\mathrm{EtOH}, \mathrm{H}_{2} \mathrm{O}$, and IPM.

\section{Discussion}

The way of drugs in a topical formulation to pass through the skin is governed by the partition (mainly between dose solution and SC) and diffusion processes through the different layers constituting the heterogeneous skin. Apparent 
FIG. 3. Diffusion (left) and distribution (right) coefficient of caffeine in the gels $(n=3$, mean \pm SEM).

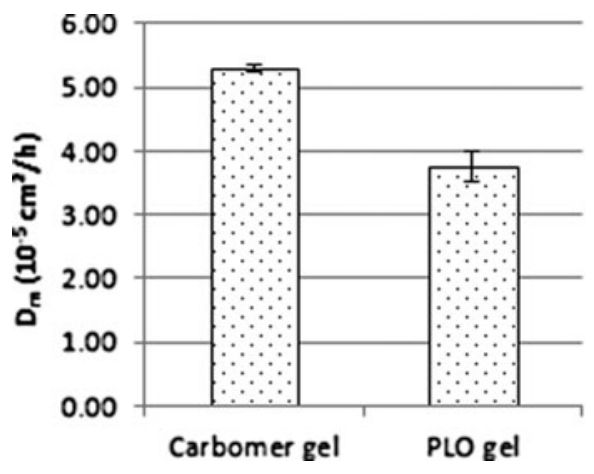

transdermal parameters reflecting these two processes (i.e., partition coefficient $K_{m}$ and diffusion coefficient $D_{m}$ ) can be experimentally determined. Hansen et al. ${ }^{12}$ collected the complete data set for caffeine (molecular weight $=194.2 \mathrm{~g} / \mathrm{mol}$, $\log \mathrm{K}_{\mathrm{oct} / \mathrm{H} 2 \mathrm{O}}=-0.083, \mathrm{pKa}=1.39$ ). From aqueous donor solutions, caffeine shows a clear partitioning into the SC. Caffeine prefers to stay in the SC environment in contrast to the more hydrophilic viable skin layers in which caffeine does not bind to the proteins present in these layers. ${ }^{12}$ This is consistent with the low-affinity binding of caffeine for human serum albumin. ${ }^{13}$ However, caffeine can reach the systemic circulation after topical application. In vivo studies with human volunteers using the follicle closure technique have shown that caffeine (formulated as $2.5 \%(\mathrm{~m} / \mathrm{V})$ caffeine, $30 \%$ ethanol, and $70 \%$ PG) reached the blood after only 5 minutes through the hair follicle pathway. ${ }^{14,15}$

In this study, the influence of two gel formulations on the transdermal penetration characteristics of caffeine was investigated. ${ }^{16}$ The Pluronic ${ }^{\circledR}$ lecithin organo gel is a phospholipidic polymeric topical gel, recently developed and marketed for transdermal applications, claiming enhanced skin permeation and absorption of drugs ${ }^{17,18}$ with lecithin acting as a penetration enhancer on the skin. The carbomer gel is a classic base, which often contains a high percentage of ethanol as well as PG, well-known skin penetration enhancers. Ethanol can cause an increased permeation of model drugs. ${ }^{19}$ Hence, our results with the carbomer-based gel having significant caffeine penetration through the skin can be ascribed to the influence of ethanol, acting as a chemical penetration enhancer. Ethanol is considered a pharmacologically inactive compound in a topical formulation, but it increases skin per-

Table $1 . \mathrm{S}_{32^{\circ} \mathrm{C}}$ Of Caffeine in the Selected Excipients $(\operatorname{MEAN} \pm \operatorname{RSD}(\%), N=2-3)$

\begin{tabular}{|c|c|c|}
\hline Excipient & $\begin{array}{c}S_{\text {caffeine }} \\
\text { (mL excipient } \\
\text { per g caffeine) }\end{array}$ & $\begin{array}{c}\text { Ph. Eur. } \\
\text { nomenclature }\end{array}$ \\
\hline $\mathrm{EtOH}$ & $163.29 \pm 1.27$ & Slightly soluble \\
\hline PG & $84.75 \pm 0.24$ & Sparingly soluble \\
\hline DGME & $66.58 \pm 0.54$ & Sparingly soluble \\
\hline IPM & $1102.85 \pm 2.12$ & Very slightly soluble \\
\hline $\mathrm{H}_{2} \mathrm{O}$ & $33.02 \pm 2.36$ & Sparingly soluble \\
\hline $\begin{array}{l}\text { 50:50 EtOH: } \mathrm{H}_{2} \mathrm{O} \\
\quad(\mathrm{V} / \mathrm{V})\end{array}$ & $9.09 \pm 1.21$ & Freely soluble \\
\hline
\end{tabular}

meability by interacting with the SC constituents. ${ }^{20,21}$ Next to purely solubility considerations, several skin-related mechanisms of actions are described for penetration enhancers: (1) disruption of the SC lipid organization, by incorporation in the bilayer to increase the free volume for drug diffusion. (2) Interaction with keratin and creation of a less dense protein structure to make it more permeable. (3) Interactions with desmosomes to alter the cohesion between corneocytes. (4) Changing the environment in the bilayer and thereby changing the solubility properties, leading to an increase in partition coefficient. ${ }^{22}$ It is likely that penetration enhancers like ethanol work through various mechanisms. ${ }^{19,23}$

To evaluate the observed effects in the comparison of the PLO versus the carbomer gel, the penetration behavior of caffeine in different solvents was studied. Ethanol and PG are examples of solvents that can increase the drug solubility in the skin. ${ }^{19,21}$ Improvement of transdermal parameters was observed for the 50:50 EtOH: $\mathrm{H}_{2} \mathrm{O}$ formulation compared to the pure $100 \% \mathrm{EtOH}$, which confirms the penetration enhancement effect of ethanol in the presence of water on caffeine, which is a hydrophilic compound. ${ }^{24}$ From Table 2, it is observed that the $K_{p}$ for caffeine in PG is very low. PG only acts as a penetration enhancer for drugs that are more soluble in alcohol than in water. ${ }^{24}$ Caffeine is sparingly soluble in water and slightly soluble in ethanol $(96 \%){ }^{25}$ These are not the optimal conditions for PG as a penetration enhancer, consistent with the low transdermal parameters found in this study. DGME is described in the literature as a nontoxic strong solubilizing agent that can act as a penetration enhancer. ${ }^{24}$ The relatively low transdermal parameter values are probably due to the fact that DGME should be used in combination with appropriate cosolvents. ${ }^{26}$

The penetration-enhancing properties of IPM were confirmed by the high transdermal flux values of caffeine through the skin. IPM can partition into both the lipid and protein areas of the $\mathrm{skin}^{27}$ and recent studies indicate that IPM disturbs the multilamellar lipid assembly. ${ }^{28}$ Moreover, after its incorporation into the SC lipids, IPM will also extract certain SC lipids into a separate phase. These effects all lead to a liquefaction of the SC lipids, accounting at least partly for the enhancing activities of IPM. Schwarb et al. ${ }^{29}$ investigated the effect of the degree of saturation of donor solutions on in vitro silicone membranes. They found that with the increasing thermodynamic activity (supersaturation) of the applied vehicle, the in vitro diffusion coefficient across the membrane was increased. When the saturation solubility of a compound in the donor solution increases and the saturation solubility of 
Table 2. Primary Transdermal Parameters (Mean \pm RSD $(\%), n=2-3$ ) for Selected Excipients

\begin{tabular}{lccc}
\hline Condition & $K_{p}\left(10^{-4} \mathrm{~cm} / \mathrm{h}\right)$ & $D_{m}\left(10^{-4} \mathrm{~cm}^{2} / \mathrm{h}\right)$ & $K_{m}\left(10^{-2}\right)$ \\
\hline EtOH & $0.90 \pm 64.15$ & $14.40 \pm 79.65$ & $0.33 \pm 107.54$ \\
EtOH+MN & $1.34 \pm 58.71$ & $5.42 \pm 53.26$ & $0.80 \pm 11.14$ \\
PG & $1.23 \pm 70.00$ & $10.51 \pm 56.16$ & $0.34 \pm 30.70$ \\
PG+MN & $1.29 \pm 44.43$ & $19.89 \pm 30.55$ & $0.23 \pm 54.03$ \\
DGME & $0.42 \pm 38.99$ & $15.43 \pm 52.76$ & $0.09 \pm 2.91$ \\
DGME $+\mathrm{MN}$ & $0.36 \pm 59.20$ & $18.23 \pm 47.39$ & $0.07 \pm 73.66$ \\
IPM & $74.31 \pm 22.85$ & $10.74 \pm 21.98$ & $23.13 \pm 16.74$ \\
IPM+MN & $95.89 \pm 16.88$ & $8.32 \pm 36.61$ & $38.63 \pm 35.34$ \\
$\mathrm{H}_{2} \mathrm{O}$ & $3.51 \pm 19.23$ & $0.97 \pm 6.08$ \\
$\mathrm{H}_{2} \mathrm{O}+\mathrm{MN}$ & $4.84 \pm 8.83$ & $11.66 \pm 31.07$ & $1.87 \pm 30.74$ \\
$50: 50$ EtOH: $\mathrm{H}_{2} \mathrm{O}(\mathrm{V} / \mathrm{V})$ & $11.43 \pm 115.16$ & $8.70 \pm 39.64$ & $5.52 \pm 137.57$ \\
$50: 50$ EtOH: $\mathrm{H}_{2} \mathrm{O}(\mathrm{V} / \mathrm{V})+\mathrm{MN}$ & $6.24 \pm 66.89$ & $9.82 \pm 41.41$ & $2.47 \pm 101.41$ \\
\hline
\end{tabular}

$\mathrm{MN}$, miconazole nitrate.

the membrane remains constant, the partition coefficient will decrease. In this study, the caffeine concentration used was $0.4 \mathrm{mg} / \mathrm{mL}$ in IPM and $2 \mathrm{mg} / \mathrm{mL}$ in the other excipients. From Table 1, it is clear that the solubility of caffeine in IPM is the lowest, while the used concentration of $0.4 \mathrm{mg} / \mathrm{mL}$ showed the highest thermodynamic activity. This can also partly contribute to the high $\mathrm{K}_{\mathrm{p}}$ value of caffeine in IPM. It should be mentioned, however, that the uniform silicone membrane as used by Schwarb et al. ${ }^{29}$ differs from the heterogeneous skin with its different layers as used in this study.

Besides the use of excipients with chemical penetrationenhancing properties, other strategies for enhancing the skin penetration are also available, such as microemulsions, liposomal-based delivery systems, microneedles, electroporation, supersaturated formulations, iontophoresis, sonophoresis, electroporation, and the use of nano- and microparticles as carriers for drugs. $21,30,31$

\section{Conclusion}

No increase in transdermal flux of caffeine was observed with the recently developed PLO gel compared to the classic carbomer-based gel formulation. The carbomer formulation consisted of a higher percentage of ethanol, which is a wellknown penetration enhancer, and which could explain the results as supported by our excipient data. Moreover, IPM showed a significant increase in the amount of caffeine penetrated through the skin, suggesting its beneficial role in topical caffeine formulations. No overall statistical significant effect of the presence of miconazole nitrate as a penetration enhancer was observed.

\section{Acknowledgment}

The authors would like to thank Bram Baert for his technical assistance during the Franz diffusion experiment.

\section{Author Disclosure Statement}

No competing financial interests exist.

\section{References}

1. Vávrová K, Zbytovská J, Hrabálek A. Amphiphilic transdermal permeation enhancers: structure-activity relationships. Curr Med Chem. 2005;12:2273-2291.
2. Dragoo KR, Silvers WM, Johnson KE, Gonzalez EA. Effects of caffeine-containing transdermal energy patch on aerobic and anaerobic exercise performance. Int J Exerc Sci. 2011;4:141-151.

3. Nicoli S, Colombo P, Santi P. Release and permeation kinetics of caffeine from bioadhesive transdermal films. AAPS J. 2005;7:E218-E223.

4. OECD, Guidance Document for the Conduct of Skin Absorption Studies, OECD Series on Testing and Assessment, Number 28. Paris: Organisation for Economic Co-operation and Development; 2004: p 21.

5. Baert B, Deconinck E, Van Gele M, et al. Transdermal penetration behaviour of drugs: CART-clustering, QSPR and selection of model compounds. Bioorg Med Chem. 2007;15: 6943-6955.

6. Baert B, Roche N, Burvenich C, De Spiegeleer B. Increase of the transdermal penetration of testosterone by miconazole nitrate. Arch Pharm Res. 2012;35:2163-2170.

7. Giacomoni PU, Mammone T, Teri M. Gender-linked differences in human skin. J Dermatol Sci. 2009;55:144-149.

8. Keleb E, Sharma RK, Mosa EB, Aljahwi AZ. Transdermal drug delivery system-design and evaluation. Int $\mathrm{J} A d v$ Pharm Sci. 2010;1:201-211.

9. Perrie Y, Badhan RKS, Kirby DJ, Lowry D, Mohammed AR, Ouyang D. The impact of ageing on the barriers to drug delivery. J Control Release. 2012;161:389-398.

10. De Spiegeleer B, Baert B, Vergote V, Van Dorpe S. Development of system suitability tests for in vitro skin integrity control: impedance and capacitance. The Eleventh International Perspectives in Percutaneous Penetration Conference: 26-29 March 2008; La Grande Motte, France.

11. ECETOC, Monograph Report No 20 Percutaneous Absorption. Brussels: European Centre for Ecotoxicology and Toxicology of Chemicals; 1993.

12. Hansen S, Henning A, Naegel A, et al. In-silico model of skin penetration based on experimentally determined input parameters. Part I:experimental determination of partition and diffusion coefficient. Eur J Pharm Biopharm. 2008;68:352-367.

13. Krisko A, Kveder M, Pecar S, Pifat G. A study of caffeine binding to human serum albumin. Croat Chem Acta. 2005; 78:71-77.

14. Otberg N, Patzelt A, Rasulev U, et al. The role of hair follicles in the percutaneous absorption of caffeine. Br J Clin Pharmacol. 2008;65:488-492.

15. Trauer S, Patzelt A, Otberg, N, et al. Permeation of topically applied caffeine through human skin-A comparison of in vivo and in vitro data. Br J Clin Pharmacol. 2009;68:181-186. 
16. Bock U, Haltner E. Impact of selected parameters on in vitro percutaneous absorption of marker compound caffeine. Int J Cosmetic Sci. 2012;34:361-361.

17. Lloyd A. The history of pluronic lecithin organogel: an interview with Marty Jones, BSPharm, FACA, FIACP. IJPC. 2003; 180-183.

18. Pandey M, Belgamwar V, Gattani S, Surana G, Tekade A. Pluronic lecithin organogel as a topical drug delivery system. Drug Deliv. 2010;17:38-47.

19. Williams AC, Barry BW. Penetration enhancers. Adv Drug Deliv Rev. 2004;56:603-618.

20. Zhao K, Singh J. In vitro percutaneous absorption enhancement of propanol hydrochloride trough porcine epidermis by terpenes/ethanol. J Control Release. 1999;62:359-366.

21. Moser K, Kriwet K, Naik A, Kalia YN, Guy RH. Passive skin penetration enhancement and its quantification in vitro. Eur J Pharm Biopharm. 2001;52:103-112.

22. Barry BW. Novel mechanisms and devices to enable successful transdermal drug delivery. Eur J Pharm Sci. 2001;14: 101-114.

23. Hadgraft J. Passive enhancement strategies in topical and transdermal drug delivery. Int J Pharm. 1999;184:1-6.

24. Trommer H, Neubert RHH. Overcoming the stratum corneum: the modulation of skin penetration. Skin Pharmacol Physiol. 2006;19:106-121.

25. European Pharmacopoeia 7.0. EDQM, Council of Europe, Strasbourg, France. 7th edition, volume 1, general notices 07/ 2010/1:10000.

26. Escobar-Chávez JJ, Quintanar-Guerrero D, Ganem-Quintanar A. In vivo skin permeation of sodium naproxen formulated in pluronic F-127 Gels: effect of azone and transcutol. Drug Dev Ind Pharm. 2005;31:447-454.
27. Das MK, Bhattacharya A, Ghosal SK. Effect of penetration enhancers on skin permeation of trazodone hydrochloride from matrix type transdermal formulation through mouse and human cadaver epidermis. Acta Pol Pharm. 2006;63: 535-541.

28. Engelbrecht TN, Demé B, Dobner B, Neubert RHH. Study of the influence of the penetration enhancer isopropyl myristate on the nanostructure of stratum corneum lipid model membranes using neutron diffraction and deuterium labeling. Skin Pharmacol Physiol. 2012;25:200-207.

29. Schwarb FP, Imanidis G, Smith EW, Haigh JM, Surber C. Effect of concentration and degree of saturation of topical fluocinonide formulations on in vitro membrane transport and in vivo availability on human skin. Pharm Res. 1999;16: 909-915.

30. Boonen J, Baert B, Lambert J, De Spiegeleer B. Skin penetration of silica microparticles. Pharmazie. 2011;66:463-364.

31. Choi WI, Lee JH, Kim, JY, Kim JC, Kim YH, Tae G. Efficient skin permeation of soluble proteins via flexible and functional nano-carrier. J Control Release. 2012;157:272-278.

Address correspondence to: Bart De Spiegeleer, PhD Drug Quality and Registration (DruQuaR) Group Faculty of Pharmaceutical Sciences

Ghent University

Harelbekestraat 72

Ghent B-9000

Belgium

E-mail: bart.despiegeleer@ugent.be 